\title{
Influence of Valsartan on the thermodynamics of micellization of anionic surfactant Sodium Dodecyl Sulphate
}

Abstract In this manuscript was investigated behaviour of drug valsartan by micellar media of anionic surfactant sodium dodecyl sulphate. As the method was used electrical conductivity for the determination of critical micelle concentration at different temperatures $(T=293.15$ - 313.15 K), as well as calculated thermodynamic parameters like standard Gibbs free energy, enthalpy and entropy of micellization. According to contribution of Gibbs free energy is the process of micellization primarily controlled by entropy. Solubilization of valsartan was studied in surfactant system at $298.15 \mathrm{~K}$ and physiological conditions pH 7.4 using UVspectrophotometry at different concentration range (0.001 - $0.07 \mathrm{~mol} / \mathrm{l})$ of sodium dodecyl sulphate. The solubilization of drug was observed with increasing concentration of surfactant in aqueous solution.

Keywords Valsartan-sodium dodecyl sulphate - critical micelle concentration - micellization - thermodynamics - solubilization

\section{INTRODUCTION}

Amphiphilic molecules such as surfactants may be spontaneously self-assemble in aqueous solutions to form thermodynamically stabile layers. These layers may close on themselves to form aggregates in solutions, known as micelles (Bergström, 2006; Kabir-ud-Din et al., 2010).

The driving force needed for amphiphilic molecules to spontaneously self-assemble into micelles and bilayers is usually called as hydrophobic effect. As a result, in order to decrease the hydrocarbon-water contact in an aqueous solution, the hydrophobic tails self-assemble to form liquidlike cores with the hydrophilic head groups located at the aggregated interface pointing towards the aqueous bulk solvent phase (Bergström, 2011).

The threshold concentration of amphiphilic molecules, in a solution above which micelles are formed, is defined as the critical micelle concentration (CMC). Above the CMC, the biological activity of micelle-forming solutes changes potentially. It is well known that amphiphile's molecular structure, as well as physico-chemical conditions such as
$\mathrm{pH}$, temperature, ionic strength, nature and concentration of additives and so on, changes the CMC of amphiphiles (drugs or surfactants) (Kabir-ud-Din et al., 2010).

The physico-chemical interactions of drugs with surfactant micelles can be considered as an approximation for their interactions with biological surface. One of the most important aspects associated with this phenomenon is the relative participation of hydrophobic and electrostatic interactions between the drug and surfactant molecule. This may explain the location of drug molecule in the micellar interior. The combination of intermolecular hydrophobic and hydrophilic interactions between surfactant and drug molecules is considered to affect the self-aggregation of surfactant molecules that leads to the formation of surfactant - drug molecular complexes (Kaushal et al., 2015).

An important property of micelles that has particular meaning in pharmacy is their ability to enhance the solubility of waterinsoluble molecules by a process known as solubilisation (Mohammad Amin Mir et al., 2011). 
Micellar system can solubilise poorly soluble drugs and thus increase their bioavailability and can be used as a model system for biomembrane, as well as for drug delivery vehicle and drug targeting systems. Micelles are known to have an anisotropic water distribution within their structure. Consequently, the water concentration decreases from the surface towards the core of micelle, with completely hydrophobic core. As a result, the spatial position of solubilised drug in micelle depends on its polarity: nonpolar molecules are solubilised in the micellar core, and substances with intermediate polarity are associated along the surfactant molecules in accurate intermediate positions (Göktürk et al., 2012).

Valsartan (VAL) lowers blood pressure by antagonising the renin-angiotensin-aldosterone system because it is a potent, highly selective and orally active antihypertensive drug belonging to the group of angiotensin II type 1 (AT ${ }_{1}$ ) receptor antagonists, of which it plays an integral role in hypertension. The main function of VAL in human body, as $A T_{1}$ receptor antagonists, is relaxing blood vessels and initiating them to widen, which lowers blood pressure and improves blood flow (Nalluri et al., 2012; Lee et al., 2014; Mak et al., 2015). It is used as a first-line agent to treat uncomplicated hypertension, isolated systolic hypertension and left ventricular hypertrophy, where it can lower blood pressure for 24 successive hours, which renders once-daily administration of VAL's effect for the treatment of hypertension (Antil et al., 2013; Lee et al., 2014). Unfortunately, the drug is poorly water soluble, which is responsible for its low bioavailability. The absolute oral bioavailability is determined to be $25 \%$, and as a result, it defends its therapeutic efficacy (Nalluri et al., 2012; Chadha et al., 2014). VAL differs structurally from losartan by an alkylated aminoacid that substitutes the heterocyclic moiety of compound. Its chemical title is $N-(1-O x o p e n t y l)-N-[[2 '-(1 \mathrm{H}-$ tetrazol-5-yl) [1,1'-biphenyl]-4-yl] methyl]-L-valine (Potamitis et al., 2011; Sanad \& Borai, 2014).

VAL contains, in its molecular structure, two weak acidic centres, the $\mathrm{COOH}$ group and tetrazole ring. The higher value $\left(\mathrm{pK}_{\mathrm{a}}=4.70\right)$ can be linked to the prevalent ionisation of tetrazole ring, whilst the lower value $\left(\mathrm{pK}_{\mathrm{a}}=3.60\right)$ is consequently ascribed to the prevalent ionisation of $\mathrm{COOH}$ group. The ionization profile of VAL shows that at $\mathrm{pH}<1.60$, this drug exists almost specifically in the undissociated form, and at $\mathrm{pH}>6.70$ and accordingly at physiological $\mathrm{pH} 7.4$, the drug exists almost as dianion (Tosco et al., 2008).

The solubility of VAL increases in the $\mathrm{pH}$ range $4-8$, and its lipophilicity decreases in the same range. VAL is soluble in water at $25{ }^{\circ} \mathrm{C}$ to the extent of $0.18 \mathrm{~g} / \mathrm{L}$, and in buffered solutions, its solubility is increased because the dianion salt is formed. As VAL has $\mathrm{pH}$-dependent solubility, it presents to a special case in a suggested general classification system, which categorises drugs with respect to their biopharmaceutical and absorption properties (Criscione et al., 1995; Saydam \& Takka, 2007).

According to the Biopharmaceutical Classification System (BCS), VAL belongs to the special group and has been classified as Class III drug with low permeability, poor metabolism and high solubility at higher $\mathrm{pH}$ values (Siddiqui et al., 2011).

The aim of this work was to study the thermodynamics of micellisation of anionic surfactant sodium dodecyl sulphate (SDS) with VAL and the solubilisation of VAL by micellar media of SDS (Fig. 1). We have discussed how drug does behave in this solution and also we have estimated and analysed various thermodynamic parameters, for example, Gibbs free energy, enthalpy and entropy of micellisation.

\section{MATERIALS AND METHODS}

\section{Materials}

The drug VAL with molecular weight $\left(M_{r}=435.52\right)$ from the group of sartans was obtained from Huahai Pharmaceuticals Co, Ltd., China, and anionic surfactant SDS with molecular weight $\left(M_{r}=288.38\right)$ was purchased from Sigma-Aldrich ChemieGmbH,Steinheim, Germany.Phosphatebuffer solution that consisted of potassium dihydrogen phosphate $\left(\mathrm{KH}_{2} \mathrm{PO}_{4^{\prime}}\right.$ $\left.M_{r}=136.09\right)$ and sodium phosphate dibasic dodecahydrate $\left(\mathrm{Na}_{2} \mathrm{HPO}_{4} \cdot 12 \mathrm{H}_{2} \mathrm{O}, \mathrm{M}_{\mathrm{r}}=358.14\right)$ at physiological pH 7.4 was prepared as a solvent for single measurements. Both of them were obtained from Lachema N.P. Brno, Czech Republic. The acidity of VAL solutions was adjusted by addition of $\mathrm{NaOH}$ (c $=0.5 \mathrm{~mol} / \mathrm{l})$.

\section{Methods and apparatus}

Stock phosphate buffer solution was prepared according to Pharmacopoeia Bohemoslovaca (PhBs, IV.Ed.) in concentration of $0.003 \mathrm{~mol} / \mathrm{l}$ for all measurements, and the $\mathrm{pH}$ value of the solution was determined and regulated potentiometrically (Inolab-pH meter, Germany) to the desired pH value 7.4.

Conductivity measurements of CMC in the presence of VAL and SDS were carried on digital conductivity meter WTW inoLab 720i (Switzerland) with thermostat Julabo ED 4 (Germany) and were based on the conductivity change (K) with surfactant concentration. This instrument can measure in the range from $0.01 \mu \mathrm{S}$ to $199.9 \mathrm{mS}$. The electrode was coated with platinum black to avoid the polarisation effect. The conductivity measurements were performed at temperature range of 293.15-313.15 K with temperature stability of $\pm 0.1 \mathrm{C}$. For the conductivity measurements, solutions of $\operatorname{SDS}(c=0.014 \mathrm{~mol} / \mathrm{l})$ with VAL $(c=0.0005 \mathrm{~mol} / \mathrm{l})$ in phosphate buffer solution were prepared and then poured into $25-\mathrm{ml}$ calibrated flask. Experiment was initiated to measure herewith solution and the subsequent concentrated solutions were obtained by adding previously prepared VAL stock solution $(c=0.0005 \mathrm{~mol} / \mathrm{l})$. After ensuring thorough mixing and temperature equilibration, the specific conductance was measured.

The calibration curve of VAL was measured using UV spectrophotometric method by UV-1800 Spectrophotometer (Japan). The stock solution of VAL was dissolved using 
<smiles>CCCCC(=O)N(Cc1ccc(-c2ccccc2-c2nn[nH]n2)cc1)C(C(=O)O)C(C)C</smiles>

(a)

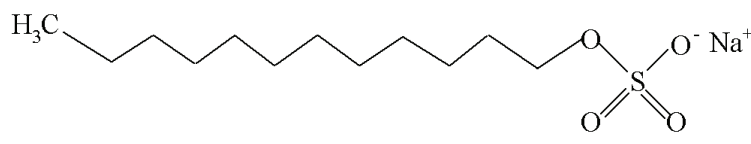

(b)

Figure 1. Structure of (a) valsartan and (b) sodium dodecyl sulphate

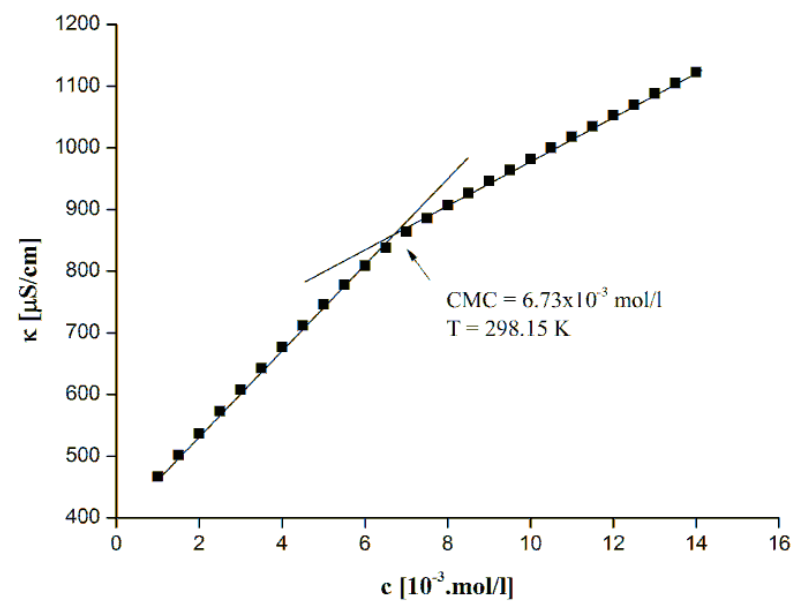

Figure 2. Plot of conductivity versus concentration of SDS in the presence of VAL at $298.15 \mathrm{~K}$. CMC, critical micelle concentration; SDS, sodium dodecyl sulphate; VAL, valsartan

ultrasound Sonorec Digitec Bandelin DT 31H (Germany) and adjusted to $\mathrm{pH}$ value of 7.4. A different amounts of volume 0.2 , $0.3,0.4,0.5,0.6,0.7,0.8,0.9,1.0$ and $1.1 \mathrm{ml}$ of VAL stock solution ( $c=0.0005 \mathrm{~mol} / \mathrm{l}$ ) were pipetted into $10-\mathrm{ml}$ calibrated flasks and subsequently diluted to volume with buffer solution. The calibration graph was measured at $\lambda_{\max }=249 \mathrm{~nm}$ for VAL.

Through the studies of solubilisation, we used an excess amount of the studied VAL (constant concentration, $\mathrm{c}=0.055 \mathrm{~mol} / \mathrm{l})$ in the presence of SDS surfactant solutions in the concentration range of $0.001-0.07 \mathrm{~mol} / \mathrm{l}(0.028-2.02 \% \mathrm{w} / \mathrm{v})$.
The prepared solutions, with excess amount of VAL and various concentrations of SDS, in a medium of buffer solutions, were added to Erlenmeyer flasks. The samples were then succussed in a shaker Kavalier LT3 (Czech Republic) at $298.15 \mathrm{~K}$ for $8 \mathrm{~h}$. After this period, the samples were filtered and the concentration of solubilisation of VAL was determined by UV spectrophotometry. The samples were prepared in phosphate buffer solution and diluted further so that the maximum absorbance may not have exceeded 1 and the Lambert-Beer law may have been obeyed. All the experiments were repeated twice.

\section{RESULTS AND DISCUSSION}

\section{Electrical conductivity measurements}

The electrical conductivity of the SDS-VAL in aqueous solutions at different temperatures (293.15-313.15 K) were measured to characterise the thermodynamic properties of micellisation. Figure 2 shows the variation of electrical conductivity versus the concentration of SDS-VAL system at 298.15 K. The experimental results fit into two straight lines with a substantial change in slope. The break point of the curve corresponds to the CMC. The CMC values at different temperatures are summarised in Table 1.

\section{Calculation of thermodynamic parameters}

The temperature dependence of InCMC (Fig. 3) was fitted to the function of the second-degree polynomial: InCMC = $\mathrm{A}+\mathrm{B} . T+\mathrm{C} . T^{2}$, which was used to determine the values of $\mathrm{A}$, $B$ and $C$. The obtained results were $A=6.80617 \pm 7.09248$, $B=-0.08123 \pm 0.04681$ and $C=0.0001397 \pm 0.0000772034$. The fit was quite less good with the coefficient of determination 0.937 .

Regarding our study, the minimum of the curve is close to $290.65 \mathrm{~K}$ according to the fit. For ionic and amphoteric surfactants, micellisation is affected by the temperature, as the hydrophobic and head group interactions change with temperature. Accordingly, CMC versus temperature studies have been performed to obtain information on these interactions (Goto et al., 1985). For ionic surfactants in an aqueous solution, the CMC decreases to a certain minimum value and then increases, displaying a U-shaped behaviour (Lee, 1995; Rosen, 2004). In nonionic surfactants, CMC decreases as the temperature is increased. This is due to an increase in the destruction of hydrogen bonds between water molecules and hydrophilic groups of the surfactants. The log CMC versus $1 / \mathrm{T}$ plot is nearly linear (Hall, 1967). However, other studies show something else, the nonionic surfactants such as polyoxyethylene glycol monoether in an aqueous solution exhibited a minimum in the CMC-temperature curve. The temperature of the minimum in the CMC-temperature curve is around $323.15 \mathrm{~K}$ and increases as the chain length of the oxyethylene increases. Though, most of the previous 
Table 1. Critical micelle concentration (CMC), enthalpy of micellisation $\left(\Delta H_{m}^{0}\right)$, Gibbs free energy of micellisation ( $\left.\Delta G_{m}^{0}\right)$, entropy of micellisation $\left(\Delta S_{m}^{0}\right)$, degree of counterion binding $(\alpha)$ and degree of dissociation $(\beta)$ for aqueous solutions of SDS in the presence of $V A L$ at different temperatures

\begin{tabular}{|c|c|c|c|c|c|c|c|c|}
\hline $\begin{array}{c}T \\
{[\mathrm{~K}]}\end{array}$ & $\begin{array}{c}\text { CMCa.10 } \\
{[\mathrm{mol} / \mathrm{l}]}\end{array}$ & $\begin{array}{c}\text { CMC.10 }^{3} \\
{[\mathrm{~mol} / \mathrm{l}]}\end{array}$ & $\begin{array}{c}\Delta G_{m}^{0} \\
{[\mathrm{~kJ} / \mathrm{mol}]}\end{array}$ & $\begin{array}{c}\Delta H_{m}^{0} \\
{[\mathrm{~kJ} / \mathrm{mol}]}\end{array}$ & $\begin{array}{c}\Delta S_{m}^{0} \\
{[\mathrm{~kJ} / \mathrm{mol} . \mathrm{K}]}\end{array}$ & $\begin{array}{c}-\boldsymbol{T} \Delta S_{m}^{0} \\
{[\mathrm{~kJ} / \mathrm{mol}]}\end{array}$ & $a$ & $\beta$ \\
\hline 293.15 & 7.94 & 6.76 & -18.27 & -1.33 & 0.057 & -16.93 & 0.489 & 0.511 \\
\hline 298.15 & 8.05 & 6.73 & -18.13 & -2.24 & 0.053 & -15.88 & 0.465 & 0.535 \\
\hline 303.15 & 8.50 & 6.91 & -18.53 & -3.91 & 0.048 & -14.61 & 0.477 & 0.523 \\
\hline 308.15 & 8.97 & 7.04 & -18.60 & -7.40 & 0.036 & -11.20 & 0.449 & 0.551 \\
\hline 313.15 & 9.57 & 7.21 & -18.64 & -9.28 & 0.029 & -9.36 & 0.440 & 0.560 \\
\hline
\end{tabular}

${ }^{a}$ Refs - CMC of SDS in doubly distilled water (Shah et al., 2001)

experiments have been carried out only up to temperature $318.15 \mathrm{~K}$. It is fair to assume that $318.15 \mathrm{~K}$ is too low to observe the minimum CMC behaviour in the CMC-temperature curve (Chen et al., 1998). The observed process of micellisation and the value of CMC depending on temperature are a complicated system, because the increase in temperature causes dehydration of hydrophilic and also hydrophobic parts. Dehydration of hydrophobic parts favours micellisation because it causes an increase in entropy, whilst dehydration of hydrophilic groups disfavours micellisation because it causes repulsion between charged hydrophilic groups. The relative size of both factors decide whether CMC will increase or decrease with temperature (Usman et al., 2013; Hanif et al., 2015). Figure 3 shows that the increase in CMC value with temperature is due to the degree of hydrophilic dehydration which is greater than that of the hydrophobic dehydration. The decrease in the value of $C M C$, in the presence of VAL in comparison to the value of CMC for pure SDS in doubly distilled water (Table 1), is mainly due to the decrease in the thickness of electric double layer surrounding the ionic head groups and due to the responsible decrease in the electrical repulsion between them in the micelle (Rangel-Yagui et al., 2005). From the above-mentioned fitting parameters, the thermodynamics magnitudes as function of temperature, Gibbs free energy $\Delta \mathrm{G}_{\mathrm{m},}^{\mathrm{o}}$ enthalpy $\Delta \mathrm{H}_{\mathrm{m} \text { ' }}^{\mathrm{o}}$ and entropy of micellisation $\Delta \mathrm{S}_{\mathrm{m}}^{0}$ have been calculated by using the following equations (Andriamainty et al., 2004; Khan et al., 2014):

$$
\Delta \mathrm{G}_{\mathrm{m}}^{\mathrm{o}}=(2-\beta) \mathrm{RT} \ln \mathrm{CMC}
$$

where $\beta$ is the degree of dissociation, $R$ is the universal gas constant having value $8.314 \mathrm{~J} \mathrm{~mol}^{-1} \mathrm{~K}^{-1}$ and $\mathrm{T}$ is the absolute temperature. $\beta$ can be calculated from the equation

$$
\beta=\frac{s_{2}}{s_{1}}
$$

where $S_{1}$ and $S_{2}$ are the slopes in the pre-micellar and postmicellar phases, respectively.

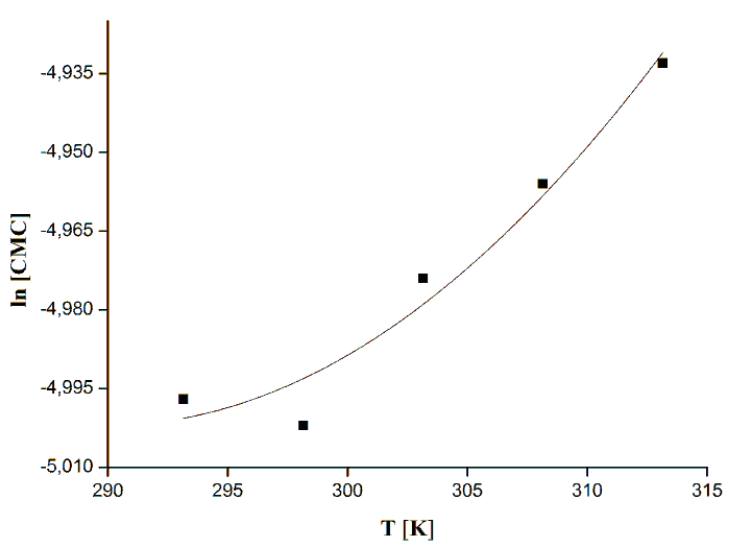

Figure 3. Plot of InCMC versus temperature of SDS in the presence of $V A L$

The degree of counterion binding a can be expressed as

$\alpha=1-\beta$

The enthalpy of micellisation is defined by the equation

$\Delta \mathrm{H}_{\mathrm{m}}^{\mathrm{o}}=-(2-\beta) \mathrm{RT}^{2}\left[\frac{\partial(\operatorname{lnCMC})}{\partial \mathrm{T}}\right]$

and the entropy contribution of micellisation can be calculated as follows:

$\Delta \mathrm{S}_{\mathrm{m}}^{\mathrm{o}}=\frac{\left(\Delta \mathrm{H}_{\mathrm{m}}^{\mathrm{o}}-\Delta \mathrm{G}_{\mathrm{m}}^{\mathrm{o}}\right)}{\mathrm{T}}$

Different parameters calculated from electrical conductivity are given in Table 1 as Gibbs free energy $\left(\Delta \mathrm{G}_{\mathrm{m}}^{\mathrm{o}}\right)$, enthalpy $\left(\Delta \mathrm{H}_{\mathrm{m}}^{\mathrm{o}}\right)$, entropy $\left(\Delta \mathrm{S}_{\mathrm{m}}^{\mathrm{o}}\right)$ of micellisation, degree of dissociation $(\beta)$ and degree of counterion binding (a) for solutions of SDSVAL system at different temperatures.

As shown in Table 1, the values of $\Delta \mathrm{G}_{\mathrm{m}}^{\mathrm{o}}$ are negative, and with increasing temperature, there is almost no change. More significant in values is the depression of standard molar enthalpy $\Delta \mathrm{H}_{\mathrm{m}}^{0}$. It is due to the spontaneous process of micellisation. 


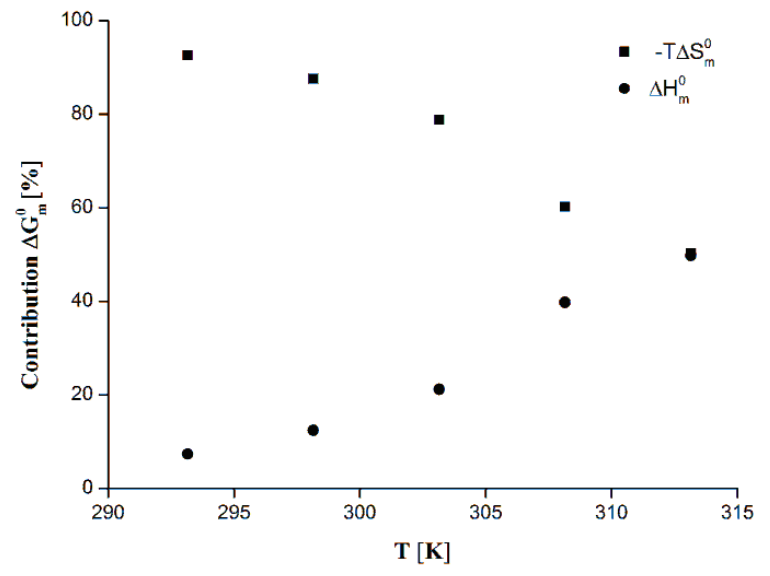

Figure 4. Contribution of enthalpy $(\bullet)$ and entropy $-T(\mathbf{\square})$ to the Gibbs free energy of micellisation for SDS in the presence of VAL

The $\Delta \mathrm{H}_{\mathrm{m}}^{0}$ is the sum of change in enthalpies arising from hydrophobic interactions, electrostatic interactions, hydration of polar head groups and counterion binding to micelles (Usman et al., 2013). The values of negative enthalpy indicate that the London dispersion interactions represent the major attractive force in the micelle formation (Del Rio et al., 1995). With increasing temperature, the enthalpy contribution to free energy increases, whereas the entropic contribution decreases as depicted in Fig. 4. The values of $\left|\Delta \mathrm{H}_{\mathrm{m}}^{\mathrm{o}}\right|$ are lower than those of $\left|-\mathrm{T} \Delta \mathrm{S}_{\mathrm{m}}^{\mathrm{o}}\right|$. It suggests that the process of micellisation is primary driven to gain the entropy. The decrease in $\Delta \mathrm{S}_{\mathrm{m}}^{0}$ values with temperature is due to the decrease in the degree of hydrophobic groups hydration at high temperature (Usman et al., 2013). It indicates that the process of micellisation is reduced at higher temperature and the micellisation inclined to be enthalpy controlled with the rise in temperature, because at high temperature, the kinetic energy of VAL and SDS molecules is higher and the probability of association of these molecules to form the complex is less. The negative values of $\Delta \mathrm{H}_{\mathrm{m}}^{\circ}$ and positive values of $\Delta \mathrm{S}_{\mathrm{m}}^{\circ}$ are probably caused by the flexible structure of SDS, which makes the micellisation easily (Usman et al., 2013).

In the following part of work, we examined solubilisation of in the presence of anionic surfactant SDS. Solubilisation is generally used as an alternative method for the dissolution of poorly soluble drugs, depending on the drug hydrophobicity. The solubilisation effect of drugs can occur in the inner core of micelle, on the surface of micelle or at an intermediate location in the palisade layer (Seedher \& Kanojia, 2008).

For the experiments of VAL solubility, the concentration of SDS in range $0.001-0.07 \mathrm{~mol} / \mathrm{l}$ at temperature $298.15 \mathrm{~K}$ was selected and the concentration of drug was kept constant $(0.055 \mathrm{~mol} / \mathrm{l})$. All the samples were determined spectrophotometrically (Table 2 ) and they refer to data of VAL solubility. The parameters of solubility $S$ (in $\mathrm{mg} / \mathrm{ml}$ ) were acquired from calibration curve of VAL, which is shown in Fig. 5 (a). As can be seen from Table 2 and Fig. 5 (b) for SDS-
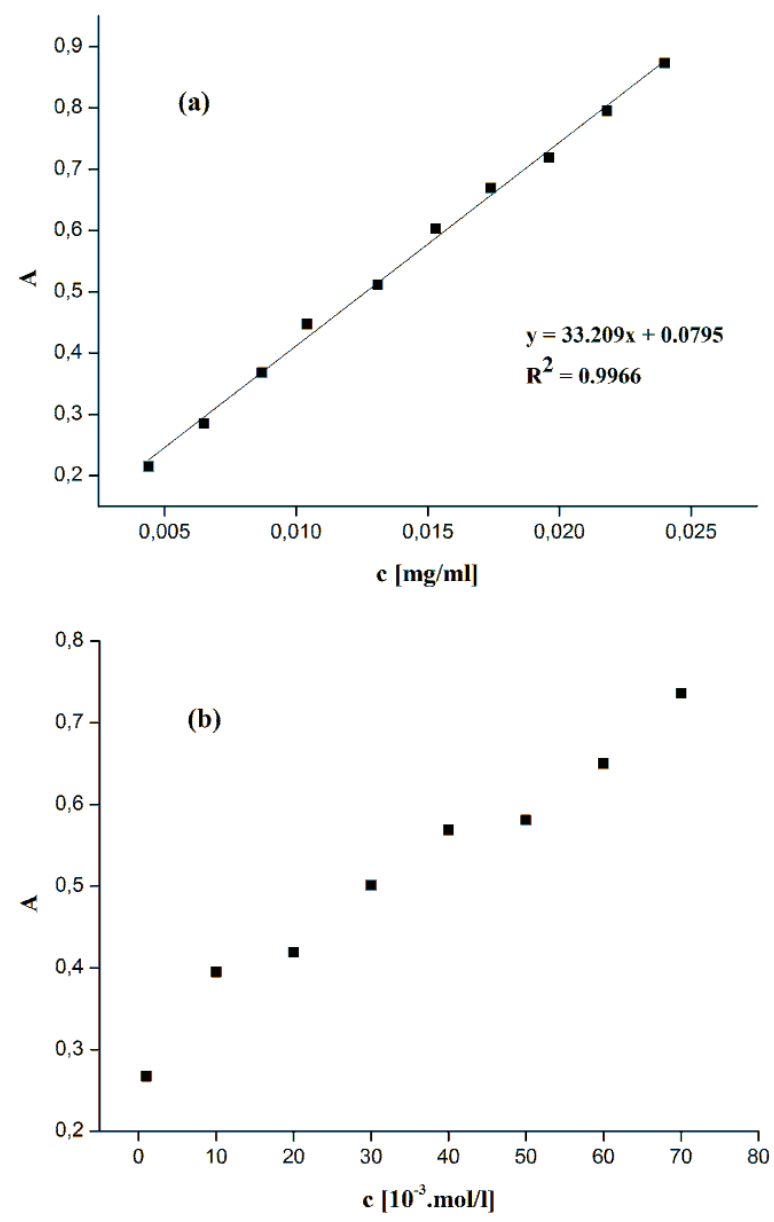

Figure 5. (a) Calibration curve of valsartan in buffer solution. (b) Plot of absorbance versus concentration of SDS in the presence of VAL $(0.055 \mathrm{~mol} / \mathrm{l})$

VAL system, the increase in drug solubility is observed for surfactant concentration above CMC and can be supposed that the micellar solubilisation is taking place.

\section{CONCLUSION}

The results obtained from conductivity enabled us to conclude that the interaction of VAL molecule with SDS and its micellisation are spontaneous processes. The values of $\Delta \mathrm{G}_{\mathrm{m}}^{\mathrm{o}}$ are negative in all considered temperatures. This shows that the micellisation process is spontaneous in the studied temperature range. Depression of standard molar enthalpy $\Delta H_{m}^{o}$ is more significant compared with $\Delta G_{m}^{o}$ values. It means that the micellisation process becomes more exothermic with increasing temperature. The process of SDS-VAL system micellisation is entropy driven in the studied temperature interval.

\section{Conflicts of interest: none.}


Table 2. Solubilisation of VAL $(0.055 \mathrm{~mol} / \mathrm{l})$ in the presence of various concentrations of SDS

\begin{tabular}{|c|c|c|c|c|}
\hline $\begin{array}{c}\text { Concentration of SDS } \\
{[\mathrm{mmol} / \mathrm{l}]}\end{array}$ & $\begin{array}{c}\boldsymbol{A} \\
{\left[\lambda_{\max }=249 \mathrm{~nm}\right]}\end{array}$ & $\begin{array}{c}\boldsymbol{S} \\
{[\mathrm{mg} / \mathrm{ml}]}\end{array}$ & $\begin{array}{c}\boldsymbol{S} \\
{[\mathrm{mmol} / \mathrm{l}]}\end{array}$ & Log S \\
\hline 1 & 0.267 & 0.564 & 1.296 & 0.113 \\
\hline 10 & 0.395 & 1.900 & 4.363 & 0.640 \\
\hline 20 & 0.419 & 3.067 & 7.042 & 0.869 \\
\hline 30 & 0.501 & 4.442 & 10.201 & 1.009 \\
\hline 40 & 0.569 & 5.896 & 13.539 & 1.132 \\
\hline 50 & 0.581 & 7.551 & 17.338 & 1.239 \\
\hline 60 & 0.650 & 8.590 & 19.724 & 1.295 \\
\hline 70 & 0.736 & 9.886 & 22.698 & 1.356 \\
\hline
\end{tabular}

VAL, valsartan; SDS, sodium dodecyl sulphate; S, solubility

\section{References}

[1] Andriamainty F,ČižmárikJ, Holíková M. Study of local anaesthetics. Part 167. Micellization of local anaesthetic heptacainium chloride in aqueous electrolyte solution. Acta Fac. Pharm. Univ. Comen. 2004;51:38-44.

[2] Antil P, Kaushik D, Jain G, Srinivas KS, Thakur I. UPLC Method for Simultaneous Determination of Valsartan \& Hydrochlorothiazide in Drug Products. J. Chrom. Sep. Techn. 2013;4(5):1-5.

[3] Bergström LM. Model calculations of the spontaneous curvature, mean and Gaussian bending constants for a thermodynamically open surfactant film. J. Colloid Interface Sci. 2006;293:181-193.

[4] Bergström LM. Thermodynamics of Self-Assembly. Application of Thermodynamics to Biological and Materials Science. 2011;289314.

[5] Criscione L, Bradley WA, Bühlmayer $P$ et al. Valsartan: Preclinical and Clinical Profile of an Antihypertensive Angiotensin-II Antagonist. Cardiovas. Drug Rev. 1995;13(3):230-250.

[6] Del Rio JM, Pombo C, Prieto G, Mosquera V, Sarmiento F. Effect of Temperature and Alkyl Chain Length on the Micellar Properties of n-Alkyltrimethylammonium Bromides in a Low pH Medium. J. Colloid Interface Sci. 1995;172: 137-141.

[7] Goto A, Takemoto M, Endo F. A Thermodynamic Study on Micellization of Nonionic Surfactant in Water and in Water-Ethanol Mixture by Gel Filtration. Bull. Chem. Soc. Jpn. 1985;58:247-251.

[8] Göktürk S, Çalişkan E, Talman RY, Var U. A Study on Solubilization of Poorly Soluble Drugs by Cyclodextrins and Micelles: Complexation and Binding Characteristics of Sulfamethoxazole and Trimethoprim. Scientific World J. 2012;1-12.

[9] Hall DG. Nonionic Surfactants Physical Chemistry. Marcel Dekker, New York; 1967, Chapter 13.

[10] Hanif S, Usman M, Hussain A, Rasool N, Zubair M, Rana UA. Solubilization of Benzothiazole (BNZ) by micellar media of Sodium dodecyl sulphate and Cetyltrimethylammonium bromide. J. Mol. Liq. 2015;211:7-14.
[11] Chadha R, Bala M, Arora P, Jain DVS, Pissurlenkar RRS, Coutinho EC. Valsartan inclusion by methyl- $\beta$-cyclodextrin: Thermodynamics, molecular modeling, Tween 80 effect and evaluation. Carbohydr. Polym. 2014;103:300-309.

[12] Chen L, Lin SY, Huang C. Temperature dependence of critical micelle concentration of polyoxyethylenated non-ionic surfactants. Colloids Surf. A Physicochem. Eng. Asp.1998;135:175181.

[13] Kabir-ud-Din, Khan AB, Naqvi AZ. Mixed micellization of antidepressant drug amitriptyline hydrochloride with cationic surfactants. Colloids Surf. B: Biointerfaces. 2010;80:206-212.

[14] Kaushal D, Rana DS, Syal VK, Chauhan S, Umar A. Micellar solubilization of Furosemide - Influence of cetyltrimethylammonium bromide in water-methanol mixture. J. Mol. Liq. 2015;211:761-766.

[15] Khan AB, Ali M, Dohare N, Singh P, Patel R. Micellization behaviour of the amphiphilic drug promethazine hydrochloride with 1-decyl-3-methylimidazolium chloride and its thermodynamic characteristics. J. Mol. Liq. 2014;198:341-346.

[16] Lee DJ. Enthalpy-Entropy compensation in ionic micelle information. Colloid Polym. Sci. 1995;273:539-543.

[17] Lee J, Lee H, Jang K, Lim KS, Shin D, Yu KS. Evaluation of the pharmacokinetic and pharmacodynamics drug interactions between cilnidipine and valsartan, in healthy volunteers. Drug Des. Dev. Ther. 2014;8:1781-1788.

[18] Mak WY, Tan SS, Wong JW et al. Bioequivalence Study of Two Valsartan 160 mg Formulations: An Open-Label, RandomisedSequence, Single-Dose, Two-Way Crossover Study in Healthy Volunteers under Fasting Conditions. J. Bioequiv. Availab. 2015;7(4):179-183.

[19] Mir MA, Chat OA, Najar MH, Younis M, Dar AA, Rather GM. Solubilization of triphenylamine, triphenylphosphine, triphenylphosphineoxide and triphenylmethanol in single and 
binary surfactants system. J. Colloid Interface Sci. 2011;364:163169.

[20] Nalluri BN, Krishna MR, RaoTP, Crooks PA. Effect of Recrystallization on the Pharmaceutical Properties of Valsartan for Improved Therapeutic Efficacy. J. App. Pharm. Sci. 2012;2(10):126-132.

[21] Potamitis C, Chatzigeorgiou P, Siapi E et al. Interactions of the $\mathrm{AT}_{1}$ antagonist valsartan with dipalmitoyl-phosphatidylcholine bilayers. Biochim. Biophys. Acta. 2011;1808:1753-1763.

[22] Rangel-Yagui CO, Wei Ling Hsu H, Pessoa-Jr A, Costa Tavares L. Micellar solubilization of ibuprofen - influence of surfactant head groups on the extent of solubilization. Braz. J. Pharm. Sci. 2005;41(2):237-246.

[23] Rosen MJ. Surfactants and Interfacial Phenomena. 3rd Ed., John Wiley \& Sons, New York; 2004, Chapter 3.

[24] Sanad MH, Borai EH. Chromatographic Separation and Utilization of Labeled99mTc-Valsartan for Cardiac Imaging. J. Mol. Imag. Dynamic. 2014;4(1):1-4.

[25] Saydam M, Takka S. Bioavailability File: Valsartan. FABAD J. Pharm. Sci. 2007;32:185-196.
[26] Seedher N, Kanojia M. Micellar solubilization of Some Poorly Soluble Antidiabetic drugs: A Technical Note. AAPS PharmaSciTech. 2008;9(2):431-436.

[27] Shah SS, Jamroz NU, Sharif QM. Micellization parameters and electrostatic interactions in micellar solution of sodium dodecyl sulfate (SDS) at different temperatures. Colloids Surf. A Physicochem. Eng. Asp. 2001;178:199-206.

[28] Siddiqui N, Husain A, Chaudhry L, Alam MS, Mitra M, Bhasin PS. Pharmacological and Pharmaceutical Profile of Valsartan: A Review. J. App. Pharm. Sci. 2011;1(4):12-19.

[29] Tosco P, Rolando B, Fruttero R et al. Physicochemical Profiling of Sartans: A Detailed Study of lonization Constants and Distribution Coefficients. Helv. Chim. Acta. 2008;91:468-482.

[30] Usman M, Rashid MA, Mansha A, Siddiq M. Thermodynamic solution properties of pefloxacinmesylate and its interactions with organized assemblies of anionic surfactant, sodium dodecyl sulphate. Thermochim. Acta. 2013;573:18-24. 